\title{
EL PLANEAMIENTO ESTRATÉGICO DEL TURISMO ECOLÓGICO EN AMÉRICA LATINA: CASO POSADA AMAZONAS DEL PERÚ Y DEL ECUADOR
}

Cesar Sarasara Andrea*

csape@yahoo.com

\begin{abstract}
RESUMEN
En el presente trabajo se han revisado diversas investigaciones existentes a nivel nacional e internacional sobre ecoturismo. El estudio tiene como objetivo proponer el planeamiento estratégico del ecoturismo en América Latina: caso Posada Amazonas del Perú y del Ecuador.

Para la obtención de los datos se aplicó una encuesta a las comunidades nativas del Amazonas del Perú y del Ecuador. El análisis de los datos se realizó mediante el método multivariado, haciendo uso del análisis factorial con el método se ha seleccionado las variables de mayor puntuación en cada grupo; también se uso las técnicas del planeamiento estratégico.

El análisis ha permitido determinar que las comunidades nativas, no están conformes con la instalación de empresas mineras, que dañen el ecosistema y apoyan el crecimiento y creación de otras empresas de turismo ecológico. Se concluye que el planeamiento estratégico para el ecoturismo en América Latina es mediante alianzas estratégicas.
\end{abstract}

Palabras claves: Estrategia, Ecoturismo, Ecológico.

\begin{abstract}
In this work we have reviewed existing research at various national and international tourism. The study aims to propose ecological Strategic Planning Ecotourism in Latin America:case Posada Amazonas in Peru and Ecuador to obtain the data, a survey of the native communities of the Amazon of Peru and Ecuador, the analysis of data using the multivariate method, using the factor analysis method has been chosen the higher ranked variables in each group was also used strategic planning techniques. The analysis has established that the native communities are not satisfied with the installation of mining, damaging the ecosystem and support the growth and creation of other eco-tourism enterprises. It is concluded that strategic planning for ecotourism in Latin America is green through strategic partnerships.
\end{abstract}

Keywords: Strategy, Ecotourism, Ecological.

\footnotetext{
* Doctorado de Administración en la Facultad de Ciencias Administrativa de la UNMSM.
} 


\section{INTRODUCCIÓN}

En la presente investigación de "El Planeamiento Estratégico del Turismo Ecológico en América Latina: Caso Posada Amazonas del Perú y del Ecuador" se pretende diseñar un modelo que contribuya a promover el desarrollo de, ecoturismo en América Latina, sea por los Estados de Latinoamérica, por las entidades privadas a nivel de la Cuenca Amazónica con los pobladores rurales principalmente con las comunidades indígenas de la selva.

Es en este sentido que también se debe impulsar y cohesionar las fortalezas de un pueblo, las Instituciones y el Estado, para acceder y usufructuar científicamente la gran riqueza natural que existe en la región. No puede haber consenso en el país sin el acuerdo entre los principales actores y protagonistas de la problemática Latinoamericana, por eso es el reto que se plantea la actividad turística, como una de las grandes potencialidades del presente milenio.

El conocimiento que se posee sobre el desarrollo del Turismo, es fruto de la evolución del hombre que ha ido manifestando, como consecuencia del grado de avance que, en el transcurso del tiempo, ha experimentado la humanidad.

Por lo mismo, en el caso del presente estudio es necesario, puntualizar que el ecoturismo es un viaje responsable y educativo a espacios naturales y zonas rurales, en especial a zonas desatendidas y regiones subdesarrolladas, contribuye a la conservación del medio ambiente y fomenta el bienestar de la población local, es una práctica ambientalmente planificada.

El Ecoturismo, palabra de nueva actualidad, se compone del prefijo "eco" que proviene de ecología y de turismo, este último término conocido desde el siglo pasado y que a su vez proviene de economía. Atendiéndonos a la primera connotación, ecoturismo vendría a ser un "Nuevo Objeto", ya que conjuga a una de las ciencias naturales, la ecología, y por la otra, al turismo perteneciente al campo de las ciencias sociales.

En el documento de Comisión de Promociones del Perú Promperú titulado. "Situación del Ecoturismo del Perú", en mayo de 2001, con el objeto de implementar los acuerdos del Acta de Actores Clave en Ecoturismo Sostenible, elabora un Programa Nacional de Turismo Sostenible y llevar a cabo las actividades en preparación para el Año Internacional del Ecoturismo-2002.
El Consejo Nacional del Ambiente (Conam) creó y convocó el Grupo Técnico de Turismo Sostenible por iniciativa de la Promperú. Esta iniciativa constituye una de las más importantes a nivel gubernamental en la búsqueda de establecer un acuerdo multisectorial (Instituciones Públicas, Empresariales, ONG y Académicas) para el Desarrollo Armónico y Sostenible del Turismo en el Perú.

Posteriormente se adhirieron al grupo, la Sociedad Nacional de Ambiente (SNA), Conservación Internacional, Confederación de Nacionalidades Amazónicas del Perú (Conap), Redtours, ONG Pro Naturaleza y la Universidad San Ignacio de Loyola (Usil).

Para viabilizar el trabajo del grupo técnico, sus miembros acordaron constituir tres Comisiones:

1. Comisión del Plan Nacional de Turismo Sostenible, cuya coordinación está a cargo del Viceministerio de Turismo. Su objetivo es la elaboración de propuestas para el establecimiento de políticas, planes y actividades intersectoriales con relación al turismo sostenible.

2. Comisión de Eventos Celebratorios del Año Internacional del Ecoturismo - 2002, bajo la coordinación de APTAE y Promperú. Su objetivo fue organizar la Conferencia Regional Andina de Ecoturismo (realizada en Febrero 2002) así como otros eventos relacionados.

3. Comisión de Ecoturismo, cuya coordinación está a cargo de Promperú. Su objetivo es elaborar un diagnóstico situacional del ecoturismo en el Perú, que sirva como base para la posición peruana en la Cumbre Mundial de Ecoturismo, que se llevó a cabo en Quebec, Canadá en mayo del 2 002; y a su vez, enlazó con el Plan Nacional de Turismo Sostenible.

El crecimiento económico del turismo en las últimas décadas ha sido notable. Los avances en este sector no se han limitado sólo a cifras que indican un movimiento de cientos de millones de dólares anualmente, sino que también los conceptos y las propuestas en este campo han evolucionado, dando lugar a la incorporación de nuevos elementos como recursos turísticos. 


\section{MÉTODOS}

\section{Materiales y Metodología.}

En el diagnóstico sobre el nivel de conocimientos relacionado a la cultura de el planeamiento estratégico del turismo ecológico en América Latina, caso Posada Amazonas del Perú y del Ecuador, para sugerir un planeamiento estratégico del turismo ecológico en América Latina, se empleó los materiales y las metodologías que a continuación se desarrolla.

\section{Entrevistas.}

Para la materialización de la presente investigación se entrevistaron a 200 pobladores tanto de las Posadas Amazonas de Perú y de Ecuador. La aplicación de la encuesta fue mediante la entrevista directa, y en forma aleatoria, el levantamiento de la información fue para conocer la apreciación de parte de los directivos de las Comunidades Nativas respecto al manejo y los problemas en los cuales se encuentran inmersas sobre el ecoturismo.

\section{Tipo de Investigación.}

El nivel de investigación es de tipo transversal, descriptiva, correlacional, multivariado, no experimental. En la investigación, se tiene cierto control en los factores que se requieren medir, obviamente el control no es total como en una investigación experimental.

\section{Encuesta.}

En la aplicación de la encuesta se siguieron las siguientes técnicas: Observación directa, u observación activa, donde el investigador participa directamente en el grupo, describiendo, evaluando, en detalle el problema sujeto a investigación. Esta técnica ha permitido obtener información datos demográficos, conocimientos sobre ecoturismo, beneficio de las empresas de ecoturismo y participación de las comunidades nativas, y participación del gobierno en esta actividad.

El cuestionario de las preguntas se diseño en base a los objetivos de la investigación, las unidades seleccionadas para la aplicación de la encuesta se ha realizado mediante un muestreo aleatorio simple.

\section{Fichas Bibliográficas.}

Se utilizaron para el levantamiento de enfoques, teorías y planteamientos que guardan cierta atingencia con el trabajo de investigación.

\section{Diseño de la Investigación}

Se hizo la elaboración de entrevistas y encuestas para ser aplicadas a las principales autoridades de las entidades públicas que se han mencionado. Asimismo, la elaboración de entrevistas a la población de la cuenca de Madre de Dios y del Ecuador con el fin de percibir su opinión que tienen sobre estas actividades.

\section{Técnica de Recolección de Datos.}

La información se obtuvo mediante la aplicación de una encuesta, para la cual se elaboró un cuestionario sobre conocimientos acerca las características generales de los pobladores de la región y sobre el nivel de conocimientos y el impacto del ecoturismo en la zona, la que se llevo a cabo primero en la Posada Amazónica Rainforest de la región de Madre de Dios y luego en la posada de la Amazonia Ecuatoriana, además se reviso información sobre el ecoturismo en otros países de la región.

\section{Unidad de Muestreo.}

La unidad de muestreo fue el lugareño de la Posada Amazónica de la Región:

\section{Variables.}

Variable Independiente: Estrategias.

Variable Dependiente: Turismo Ecológico.

Variable intervinientes: Conocimiento sobre Turismo Ecológico.

Participación en el Ecoturismo.

Impacto del Ecoturismo en las Comunidades Nativas.

Participación del Gobierno en el Ecoturismo.

\section{RESULTADOS}

Para realizar la presente investigación se entrevistaron a 160 nativos de las Comunidades de Ese'Eja, Comunidad Nativa Infierno, de Madre de Dios, del Perú y el de las Comunidades Nativas Achuar del Alto Pastaza de Ecuador, a los que se les aplicó una encuesta dividida en cuatro secciones.

Se determina que la edad promedio de los Nativos del Perú es de 31.33 años con una desviación estándar de 11.27 años, siendo la edad mínima de 14 años y la edad máxima de 58 años, en lo que respecta al género el $75 \%$ corresponde a 
nativos varones, y el 25\% a mujeres nativas; en los encuestados nativos del Ecuador se registra una edad promedio de 34.66 años, con una desviación estándar de 9.10 años, siendo la edad mínima de 18 años y la edad máxima de 65 años, el 71\% corresponde a nativos del sexo masculino y $29 \%$ a nativas de sexo femenino.

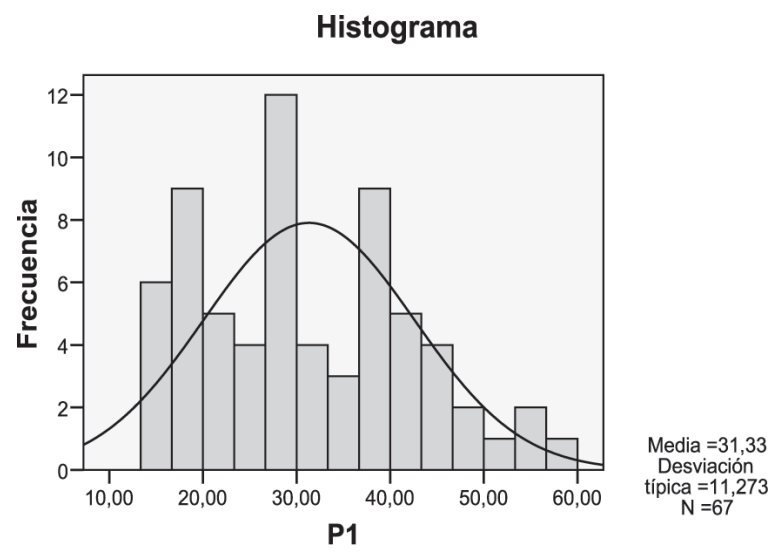

A: COMUNIDAD NATIVA DEL PERÚ

GRUPO I: CONOCIMIENTO SOBRE TURISMO ECOLÓGICO (ECOTURISMO)

Gráfico №: Registro de nativos según género

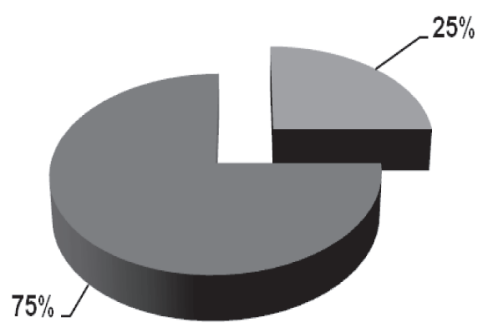

FEMENINO

MASCULINO

El mayor porcentaje de nativos encuestados corresponde al sexo masculino P5

Gráfico № : registro de nativos por ocupación

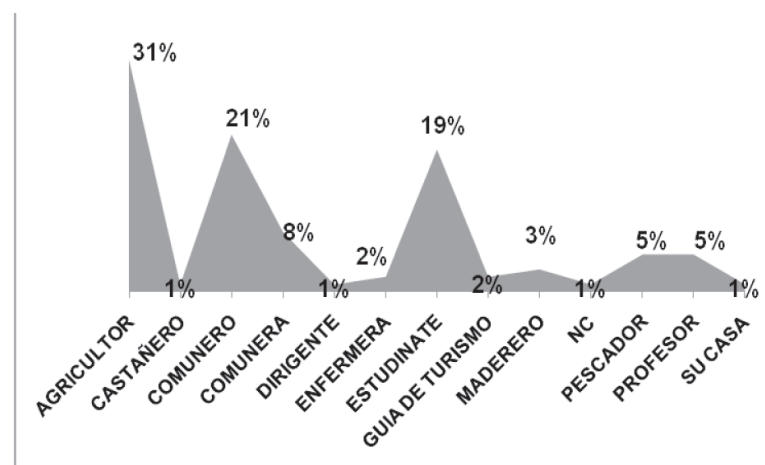

Los encuestados responden en mayor porcentaje que tienen como ocupación la agricultura, otro porcentaje significativo corresponde a nati- vos comuneros, los nativos en un alto porcentaje son estudiantes y en porcentajes mínimos se registra a nativos que son dirigentes, enfermeras, guías de turismo, maderero, y las que realizan labores en su casa.

Gráfico №: Reciben Información para Conservar el Medio Ambiente

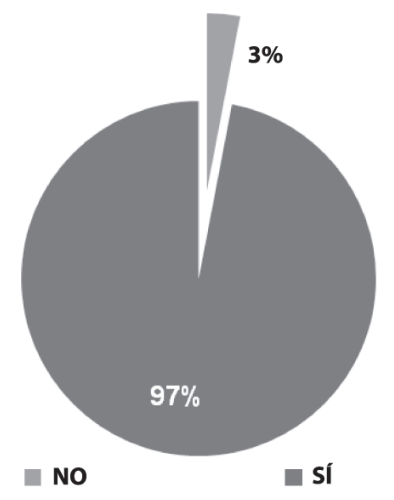

Los encuestados afirman en un porcentaje significativo que reciben información para conservar el medio ambiente.

Gráfico № : Registro de quienes brindan información

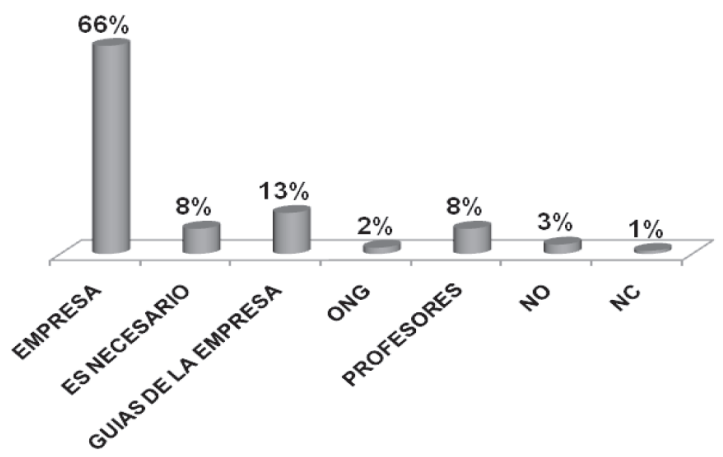

De los resultados de los encuestados se determina que un alto porcentaje de nativos recibe información sobre ecoturismo por parte de la empresa, también responden que reciben información por parte de los profesores y de las ONG.

Gráfico №: Respuestas de los nativos sobre: Considera que los empresarios realizan una buena actividad para conservar el medio ambiente:

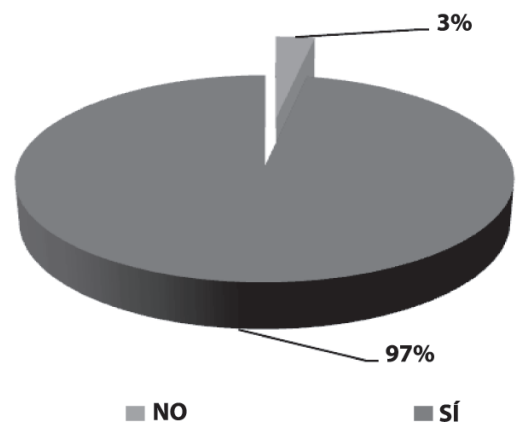


Los encuestados en un alto porcentaje muy significativo responden que los empresarios si realizan una buena actividad para conservar el medio ambiente.

\section{GRUPO II: PARTICIPACIÓN EN EL ECOTURISMO}

Gráfico №: participación de los nativos en el Ecoturismo

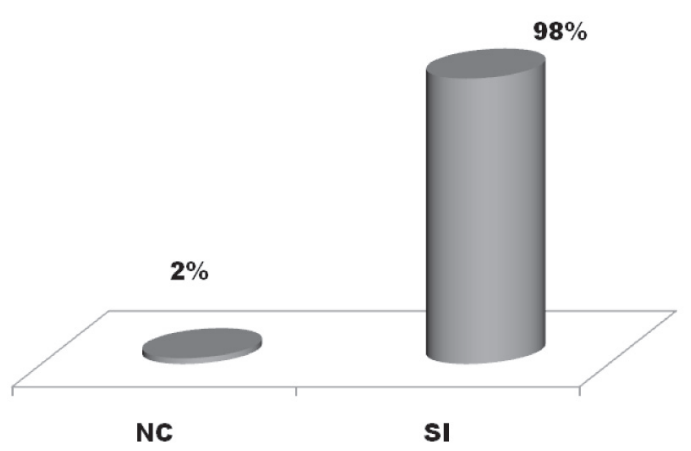

Los resultados del estudio muestran que los nativos afirman que tienen participación en las empresas de ecoturismo.

Gráfico №: Reciben algún beneficio por esta actividad

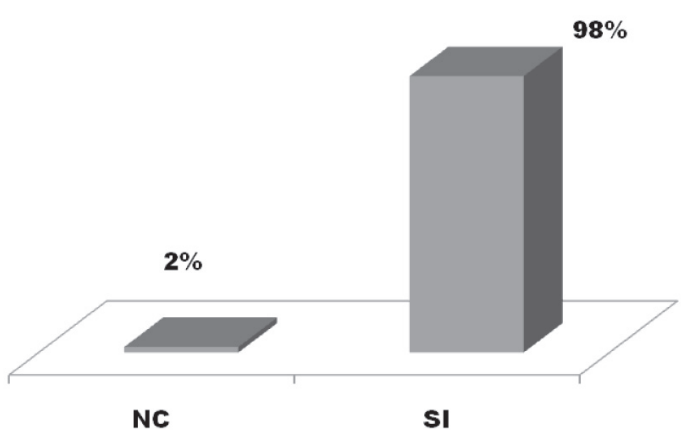

Los nativos encuestados afirman que sí reciben beneficio de la empresa de ecoturismo, un mínimo porcentaje responde que no.

Gráfico №: Tipo de beneficio que reciben los nativos por el ecoturismo

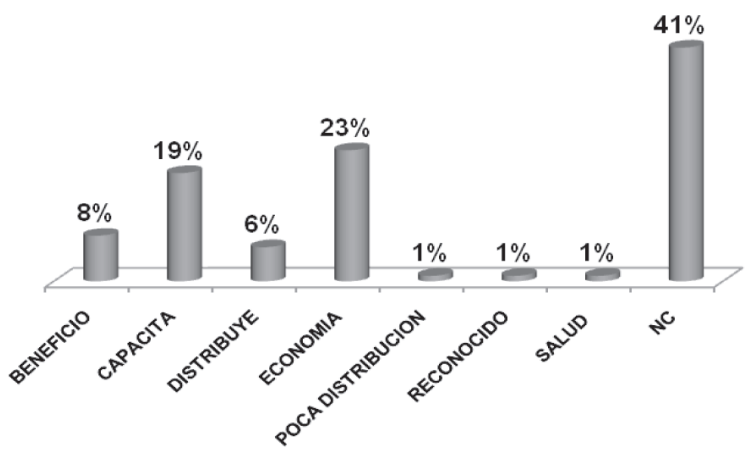

Un alto porcentaje de encuestados no responde el tipo de beneficio que reciben de la empresa de ecoturismo, porcentajes significativos afirman que reciben beneficio económico y la capacitación.

Gráfico №: Participación de la comunidad en las actividades de Ecoturismo

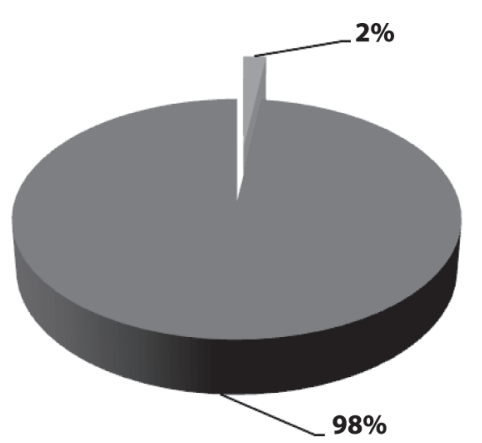

NC

SI

Los encuestados afirman en un porcentaje alto que tienen participación en las actividades del ecoturismo.

Gráfico №: La Comunidad se beneficia con la actividad de Ecoturismo

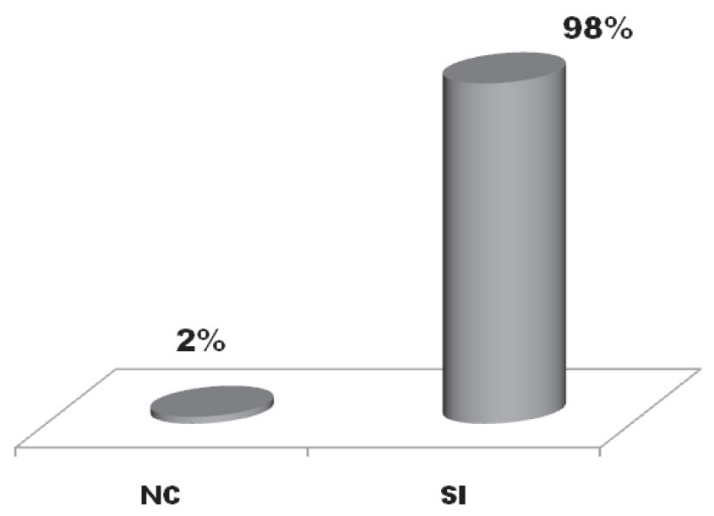

Los resultados del estudio muestran que un alto porcentaje de nativos afirman que su comunidad se beneficia con la actividad de Ecoturismo.

Gráfico №: Beneficio que recibe la comunidad

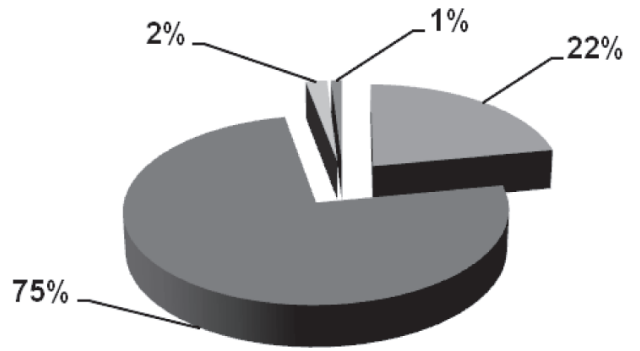

$$
\because \text { SOCIA } \because \text { SOCIO } \square \text { EMPRESARIA } \square \text { NC }
$$

Los nativos responden que reciben beneficio por que son socios de la empresa de ecoturismo. 
Gráfico №: Participación individual de los Nativos en la actividad de Ecoturismo

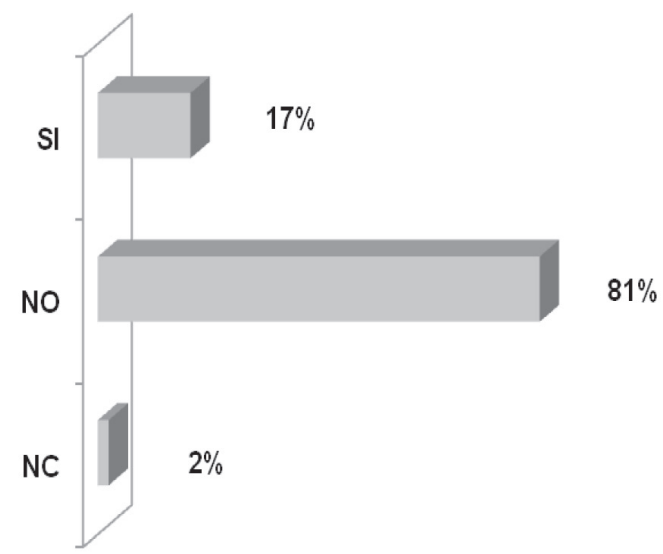

Los nativos encuestados afirman que individualmente participan en las actividades del Ecoturismo.

\section{GRUPO III: IMPACTO DEL ECOTURISMO EN LAS CO- MUNIDADES NATIVAS}

Gráfico №: Para Usted, Considera que Existe Impacto del Ecoturismo

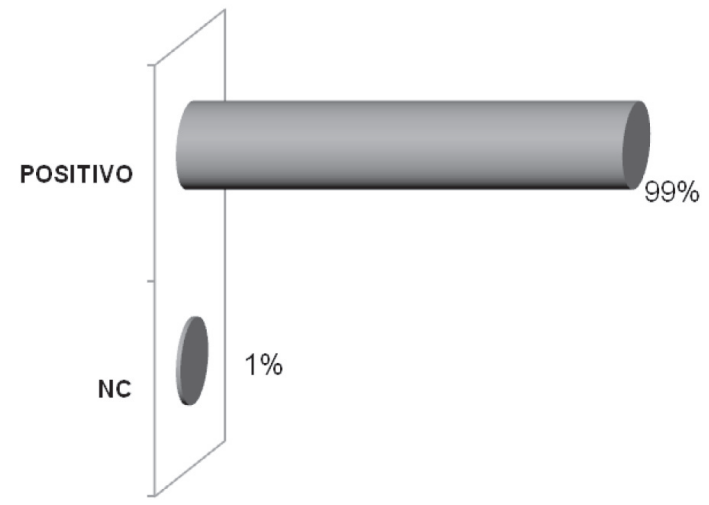

Según los encuestados, afirman en un alto porcentaje que el impacto del Ecoturismo es Positivo.

Gráfico №: Percepción sobre las Actividades del Ecoturismo

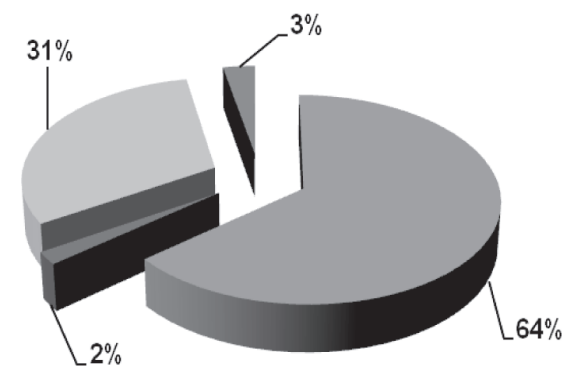

- BUENO $\quad$ ALGO BUENO MUYBUENO $\square$ NC
Los nativos encuestados aseguran en un porcentaje significativo que tienen buena percepción sobre las actividades del ecoturismo, en porcentaje mínimo afirman que la percepción es algo bueno.

\section{GRUPO IV: PARTICIPACIÓN DEL GOBIERNO EN EL ECOTURISMO}

Gráfico №: Consideran que las políticas de gobierno sobre ecoturismo favorecen a la comunidad

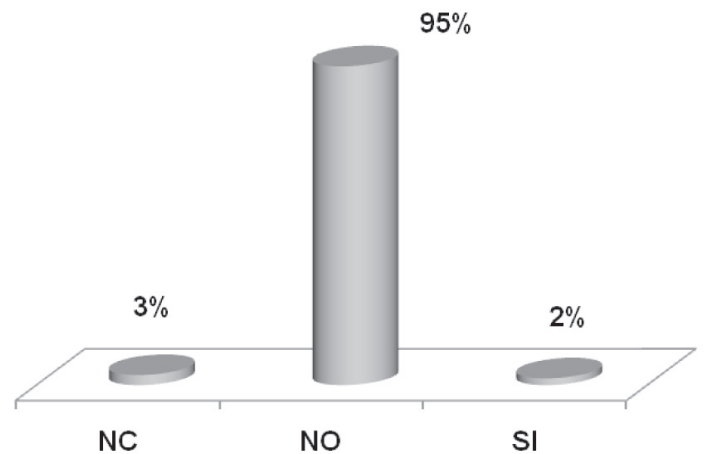

En un porcentaje muy significativo los nativos encuestados responden que las políticas de gobierno no favorecen a la comunidad.

Gráfico №: Su comunidad cuenta con servicios básicos

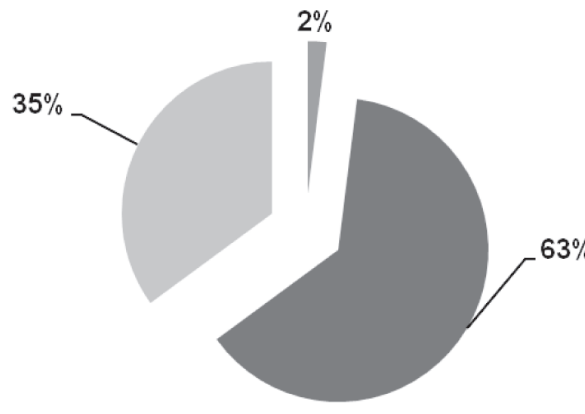

$\square$ NC $\quad$ NO $\quad$ SI

Un alto porcentaje de nativos encuestados aseguran que no cuentan con servicios básicos, en menor porcentaje responde que si cuenta con algún servicio básico.

Gráfico №: Cuenta con Servicios de Salud

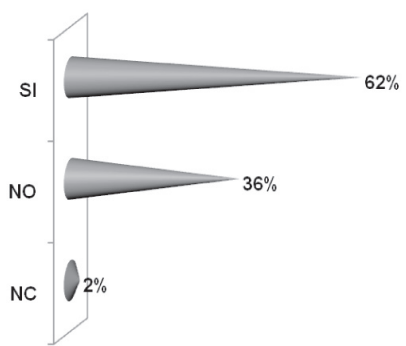


Un porcentaje alto afirma que no cuenta con servicios de salud.

Gráfico №: Cómo se atiende cuando tiene una dolencia

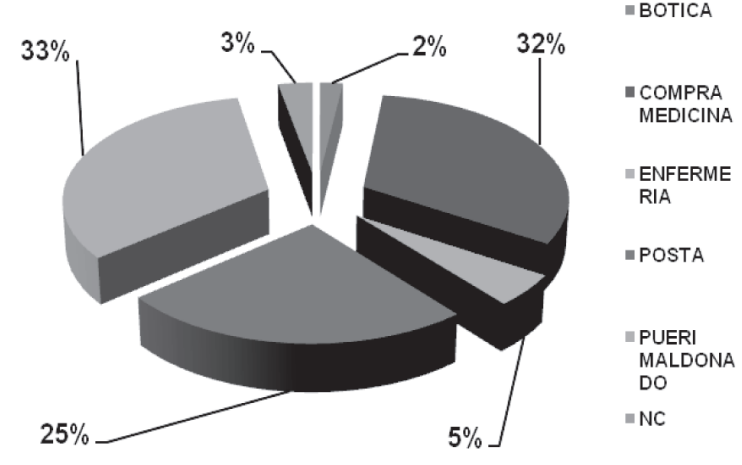

Los resultados del estudio muestran que en porcentajes significativos los nativos se atienden de sus dolencias con medicinas tradicionales y acuden a Puerto Maldonado en caso de emergencia, y en ocasiones acuden a la Posta Médica de cada lugar.
Gráfico №: Su comunidad cuenta con centros educativos

$95 \%$

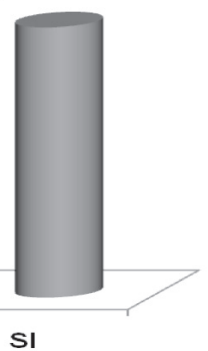

sı

Los nativos encuestados responden que cuentan con escuelas de educación primaria.

\subsection{Análisis, Interpretación y Discusión de Re- sultados}

Resultados: Análisis Factorial

Medida de adecuación Kaiser - Meyer - Olkin (kmo) para los Datos de los Cuatro Grupos del Estudio: El Planeamiento Estratégico del Turismo Ecológico en América Latina: Caso Posada Amazonas del Perú y del Ecuador.

\section{KMO y prueba de Bartlett}

\begin{tabular}{|c|c|c|}
\hline \multicolumn{2}{|c|}{$\begin{array}{l}\text { Medida de adecuación muestral de } \\
\text { Kaiser-Meyer-Olkin. }\end{array}$} & 528 \\
\hline $\begin{array}{l}\text { Prueba de esfericidad } \\
\text { de Bartlett }\end{array}$ & $\begin{array}{l}\text { Chi-cuadrado } \\
\text { aproximado }\end{array}$ & 21.389 \\
\hline & gl & 6 \\
\hline & Sig. & .002 \\
\hline
\end{tabular}

\section{a. Basado en correlaciones}

El análisis de los datos para el estudio, Planeamiento Estratégico para el Turismo Ecológico en América Latina: caso Posada Amazonas del Perú y del Ecuador, es adecuado para realizar un análisis multivariado siendo el valor para la medida de adecuación del modelo $\mathrm{KMO}=0.528$, con un nivel de significancia $=0.002$, siendo su $\mathrm{p}-$ valor menor de 0.05

ANÁLISIS FACTORIAL POR GRUPOS

\begin{tabular}{|l|l|l|}
\hline SECCIONES & KMO & SIG \\
\hline GRUPOS: I, II, III, IV, & 0.528 & 0.00 \\
\hline GRUPO I: CONOCIMIENTO SOBRE ECOTURISMO & 0.529 & 0.04 \\
\hline GRUPO II: PARTICIPACIÓN DE LOS NATIVOS EN EL ECOTURISMO & 0.501 & 0.00 \\
\hline $\begin{array}{l}\text { GRUPO III: IMPACTO DEL ECOTURISMO EN LAS COMUNIDADES } \\
\text { NATIVAS }\end{array}$ & 0.514 & 0.00 \\
\hline GRUPO IV: PARTICIPACIÓN DEL GOBIERNO EN EL ECOTURISMO & 0.629 & 0.00 \\
\hline
\end{tabular}




$$
\mathrm{P}<0.05
$$

Del análisis realizado mediante la medida de adecuación a las secciones que agrupan las variables del estudio, se observa que el Grupo IV conformado por variables (Considera que las políticas de gobierno favorecen a su comunidad, su comunidad cuenta con servicios básicos, tienen servicios de salud, su comunidad cuenta con Centros Educativos), presenta un $\mathrm{KMO}=0.629$ con un nivel de significancia de 0.00 (p - valor menor de 0.05), el Grupo I que reúne a las variables (Conocimiento sobre Ecoturismo, opinión sobre Ecoturismo, están de acuerdo con este tipo de actividad, considera que la actividad conserva el medio ambiente, reciben información para conservar el Medio Ambiente) muestra un $\mathrm{KMO}=0.529$ con un nivel de significancia 0.04 (p-valor menor de 0.05), el Grupo III, (Visión sobre el Ecoturismo, Impacto del Ecoturismo, Percepción sobre las Actividades del Ecoturismo), muestra un $\mathrm{KMO}=$ 0.514 con un $\mathrm{p}$ - valor menor de 0.05 .

El Grupo II (Participación en el Ecoturismo, Reciben algún Beneficio de esta Actividad, Tipo de Beneficio, Participación de la Comunidad en esta Actividad, la Comunidad recibe algún beneficio, participación individual en esta actividad), presentan un $\mathrm{KMO}=0.501$, con un $\mathrm{p}$ - valor menor de 0.05 ( $p=0.00)$, se determina que las variables objeto de estudio se adecuan para realizar un análisis Multivariado, siendo las cuatro secciones y el conjunto de variables en general significativas.

Tabla №: Validez del constructo mediante la prueba Análisis de componentes principales

\begin{tabular}{|l|c|c|}
\hline \multirow{2}{*}{ SECCIONES } & \multicolumn{2}{|c|}{ COMPONENTES } \\
\hline GRUPO I: CONOCIMIENTO SOBRE ECOTURISMO & 1 & 2 \\
\hline GRUPO II: PARTICIPACIÓN DE LOS NATIVOS EN EL ECOTURISMO & 0.597 & 0.184 \\
\hline GRUPO III: IMPACTO DEL ECOTURISMO EN LAS COMUNIDADES NATIVAS & 0.113 & 0.977 \\
\hline GRUPO IV: PARTICIPACIÓN DEL GOBIERNO EN EL ECOTURISMO. & 0.334 & 0.360 \\
\hline
\end{tabular}

Del conjunto de variables que conforman el estudio sobre el planeamiento estratégico del Turismo Ecológico en América Latina: Caso Posada Amazonas del Perú y del Ecuador, se determinan que el aporte de las variables al estudio, según los grupos consignados en las diferentes secciones es el 97.7 \% de aporte del Grupo II (Participación de los Nativos en el Ecoturismo) el Grupo IV (Participación del Gobierno en el Ecoturismo) aporte con el 90.2.\%, el Grupo I (Conocimiento del Ecoturismo) aporta con $59.7 \%$, siendo estos aportes significativos en la evaluación para la investigación y el Grupo III (Impacto del Ecoturismo en las Comunidades Nativas) aporta solo con $36 \%$ aportes.

Tabla 5: Análisis de las variables mediante el método de componentes principales

\begin{tabular}{|l|c|}
\hline \multicolumn{1}{|c|}{ VARIABLES } & Extracción \\
\hline V5: Conoce sobre Ecoturismo .(participación en grupo) & 0.625 \\
\hline V6: Qué opina sobre el ecoturismo .(participación en grupo) & 0.625 \\
\hline V7: Está usted de acuerdo con esta actividad. .(participación en grupo) & 0.625 \\
\hline V8: Considera que la actividad conserva el medio ambiente. .(participación en grupo) & 0.625 \\
\hline V9: Reciben información sobre conservación del medio ambiente & 0.969 \\
\hline V10: Consideras que las empresas realizan una buena actividad para conservar el medio ambiente & 0.437 \\
\hline
\end{tabular}




\begin{tabular}{|l|c|}
\hline V11: Ustedes tienen participación en el ecoturismo .(participación en grupo) & 0.923 \\
\hline V12: Recibe algún beneficio de esta actividad & 0.641 \\
\hline V13: Qué tipo de beneficio recibe Ud. & 0.992 \\
\hline V14: Su comunidad participa de esta actividad.(participación en grupo) & 0.923 \\
\hline V15: Su comunidad se beneficia con esta actividad & 0.305 \\
\hline V16: Usted individualmente participa en esta actividad. & 0.970 \\
\hline V18: Considera Ud. que el impacto del Ecoturismo es Positivo & 0.498 \\
\hline V20: Cuáles son las aspiraciones a futuro & 0.998 \\
\hline V21: Considera que las políticas del gobierno favorece a su comunidad & 0.951 \\
\hline V22: Su comunidad cuenta con servicios básicos & 0.976 \\
\hline V23: Tienen servicios de salud & 0.512 \\
\hline V25: Su comunidad cuenta con servicios educativos & 0.407 \\
\hline
\end{tabular}

Método de extracción: Análisis de Componentes principales.

Del estudio individual de las variables se determina que el aporte significativo al estudio en la primera componente es para la variable aspiraciones de los nativos a futuro con $99.8 \%$, la variable tipo de beneficio que reciben los nativos aporta con $99.2 \%$, mientras que la variable la comunidad nativa cuenta con servicios básicos aporta el $97.6 \%$, la variable participación individual en la actividad de ecosistema aporta con $97.0 \%$, reciben información del medio ambiente aporta con 96.9\%.

TABLA 6: ANÁLISIS DE VARIANZA

\begin{tabular}{|l|l|l|l|l|l|}
\hline \multicolumn{1}{|c|}{ FUENTE } & SUMA DE CUADRADOS & $\begin{array}{l}\text { GRADOS DE } \\
\text { LIBERTAD }\end{array}$ & \multicolumn{1}{|c|}{$\begin{array}{c}\text { CUADRADOS } \\
\text { MEDIOS }\end{array}$} & F & SIG \\
\hline ENTRE GRUPOS & 21.025 & 4 & 5.256 & 325.301 & 0.00 \\
\hline DENTRO DE GRUPOS & 1.535 & 95 & 0.016 & & \\
\hline TOTAL & 22.560 & 99 & & & \\
\hline
\end{tabular}

$\mathrm{R}=0.965$

La variable conocimiento sobre Ecoturismo, participación de los nativos en el Ecoturismo, Impacto del Ecoturismo en las comunidades nativas, participación del Gobierno en el Ecoturismo, influye significativamente sobre el Turismo Ecológico en América Latina, con un $\mathrm{F}=325.301$, con $\mathrm{p}<0.05$ ( $\mathrm{p}=$ 0.00 ) y con un nivel de correlación de las variables $\mathrm{R}=96.5 \%$.

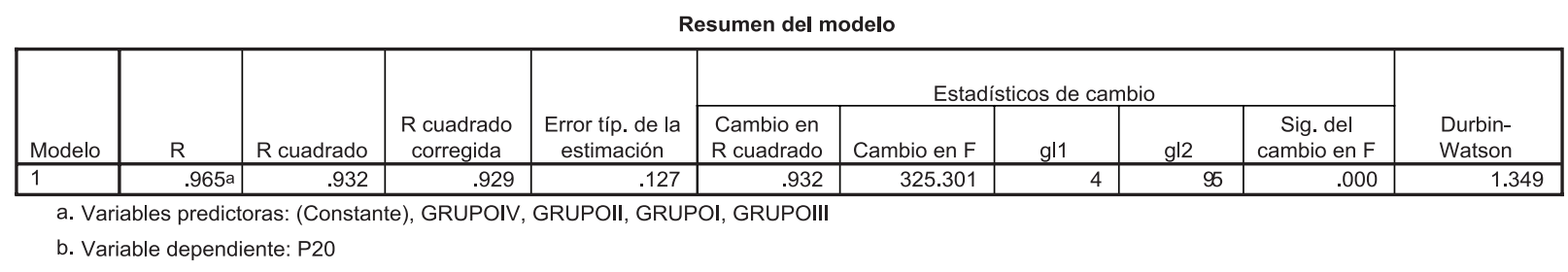




\section{DISCUSIÓN}

\section{Propuesta para la solución del problema}

5.1.1 Propuesta mediante la aplicación del método del proceso de planeamiento estratégico.

La investigación pretende demostrar que el planeamiento estratégico contribuye para fomentar la actividad económica del ecoturismo en la región protegiendo los bosques y la biodiversidad, asociado a sus pobladores, que tomen conciencia de lo que sucede con los efectos de la globalización económica y social, contaminación ambiental y mejoren su calidad de vida.

El resultado de esta investigación del planeamiento estratégico de ecoturismo será un instrumento válido para el uso y manejo de las entidades públicas, Privadas y en las fuentes cooperantes nacionales como extranjeras en la tomas de decisiones políticas en los ámbitos rurales para que contribuyan y exploten la conservación del bosque mediante alianzas estratégicas con las poblaciones locales, y les represente una oportunidad también a los aborígenes, y definan su visión de corto, mediano y largo plazo, el incremento de la fuente del empleo, conciencia sobre el deterioro ambiental y adopten por un turismo ambientalmente sano y compatible.

\subsection{Costos de Implementación de la propuesta}

\subsubsection{Económicas}

Al corregir las causas que originan el fomento de la actividad turística del desarrollo del Ecoturismo en la zona, se logrará romper la postergación política, y con la información oportuna se incrementará la captación de ingresos y con la correcta aplicación de los fondos las familias, el gobierno local tendrá mayor liquidez para sus fines, objetivos y metas. Asimismo es en este punto que se planteará la implementación de la estrategia del valor de la marca del trabajo emprendido.

\subsection{Beneficios que aporta la propuesta}

Uno de los impactos más desfavorables que una entidad donante puede causar a una empresa comunitaria es la inversión de fondos antes que exista una estructura comunitaria que permita la toma de decisiones. Los modelos verticales para la toma de decisiones, en los cuales la entidad donante ocupa el lugar más alto, inevitablemente impiden los esfuerzos de la comunidad para manejar sus propios fondos.

\subsubsection{Sociales}

Los resultados del estudio que se obtengan con relación al desarrollo del Ecoturismo, se remitirán a los gobiernos de las comunidades nativas (gobiernos comunales indígenas), gobiernos locales (municipalidades), y a los gobiernos regionales, que constituirán bases importantes para diseños de sus planes de desarrollo regional, repercutiendo en beneficio de los centros poblados, comunidades, inversores y demás organizaciones sociales

\subsubsection{Científicas}

Será dada por la sistematización de Nuevos enfoques teóricos sobre el planeamiento estratégico para el desarrollo del ecoturismo y esta información oportuna debe ser procesada por las municipalidades de la provincia de Puerto Maldonado, en el caso peruano, y el Alto Pastaza, en el caso ecuatoriano, es decir, las técnicas, de manejo ambiental de gestión empresarial ciertamente contribuirán en su aplicación práctica.

\section{CONCLUSIONES}

Iniciar un proyecto de ecoturismo comunitario vinculado a la explotación del turismo Ecológico implica la contratación de expertos tanto indígenas de concurso internacional y no indígenas con experiencia en esta temática de desarrollo comunitario, inclusive a expertos en otras disciplinas como en antropología y sociología del desarrollo, para evaluar el contexto sociocultural y la viabilidad de la implementación de una asesoría sostenida con una maduración, provocando una actividad con un enfoque conducente a una situación de largo plazo.

Es necesario que el estado asuma su rol promotor innegable en el caso de las comunidades nativas en concordancia a los artículos 88 y 89 del régimen agrario de las comunidades campesinas y nativas, de la Constitución Política del Perú. Asimismo, amos estados nacionales (Ecuador-Perú), por el gesto de su ratificación del Convenio Internacional de Trabajo número 169 , en concordancia a los artículos pertinentes que emana asumir responsabilidades en la posibilidad de implementar proyectos de desarrollo mediante políticas de buena gobernanza; la convocatoria a esta iniciativa involucra también a los demás países Latinoamericanos que han ratificado el Convenio № 169 de la OIT. 
En este sentido, damos por acertada que el ambiente social y cultural permite para generar los proyectos de ecoturismo comunitarios se inicien con la participación plena que garantice con el suficiente apoyo y energía de la misma comunidad. Para evitar las frustraciones, no se puede esperar que todos los proyectos cuenten con el favor de todos los miembros de la comunidad, porque si al comienzo cuentan con un liderazgo talentoso y creativo apoyado por la mayor parte de los residentes, es más probable que ese proyecto tenga éxito.

Las Comunidades Nativas de la Cuenca Amazónica, del Colombia, Ecuador, Bolivia, Paraguay, Venezuela, Surinam, Brasil y del Perú, que en la actualidad se les reconoce como "Pueblos Indígenas" (Convenio № 169), se encuentran aisladas, por la sociedad financiera, principalmente por aquellos sectores que provienen del capitalismo nacional e internacional; esta condición a nivel interno agrava a que se funde la desarticulación socioeconómica en el consumo descontrolado e irracional de sus recursos naturales que disponen y la oferta de sus patrimonio comunal, los Recursos Naturales entre otros, a precios no convencionales, formalización de alianzas estratégicas con muchas desventajosas, alquiler de tierras, acaparamiento de tierras comunales por familias de élites y demás problemáticas que comprometen la vida comunal en su totalidad.

\section{LITERATURA CITADA}

Alvarado Oyarce, Otoniel.2000. Elementos de la Administración General. Lima - Perú. Editorial Udegraf S.A.

Alvares Lobo, Ricardo. Sepahua II. Fundación de una Misión Católica en el Bajo Urubamba. Impresión Enotria S. A. Lima Perú 1996, 407PP.

Arnalot, José. Lo que los Achuar me han enseñado. Ediciones Abya -Yala. Quito Ecuador. 2007, 428 PP.

Avellaneda Cusaria, Alfonso. Gestión Ambiental y Planificación del Desarrollo. El Reloj Verde. Ecoe Ediciones. Impresión: Lito Perla Impresores. Bogotá, D.C., 2002, 232PP.

Bustamante Caro, Pedro y otros. Nuevo Diccionario de Sinónimos, Antónimos y Parónimos. Lima - Perú. Editorial Consorcio Editor Nuevo Perú S.A.C.
Centro Cultural José Pío Aza. Papachí Ese Eja. Impreso en los Talleres de: Grafimag S.R.L. Lima 2006, 880 PP.

Chumap Lucia, Aurelio y Manuel García - Rendueles. Duik Múun. Universo Mítico de los Aguaruna. Tomo I y II. Impreso en el Centro de Proyección Cristiana. Lima Perú 1979.

COICA. Coordinadora de la Organizaciones Indígenas de la Cuenca Amazónica. Diversidad y Derechos de los Pueblos Indígenas. Manual de Capacitación de base. Imprenta de Coica. Quito-Ecuador 1998, 33PP.

Collazos Cerrón, Jesús. 2005. Manual de Evaluación ambiental de proyectos. Lima-Perú Editorial San Marcos.

CONAP. Confederación de Nacionalidades Amazónicas del Perú. Explotación Minera a Pequeña Escala Entre los Pueblos Harakmbut y Awajún de la Amazonia Peruana. Impresión en los Talleres de Códice Ediciones S.A.C. Lima - Perú 2007, 192 PP.

CONAP. Confederación de Nacionalidades Amazónicas del Perú. La Cultura Ancestral Matsigenka: Respuesta a la Modernidad del siglo XXI. Impresión Grafica Biblos S.A. Lima, Perú. 2006, 142 PP.

Constitución Política del Perú. Impresión en los Talleres Gráficos de Ediciones Cultura Peruana. Lima 2003, 95 PP.

Dajos Roger. Tratado de Ecología. Ediciones Mundi-Prensa 2- Edición Impreso en España. Madrid 200, 600 PP.

Descola Philippe. Las Selva Culta. Simbolismo y Praxis en la Ecología de los Achuar. Tercera Edición. Ediciones Abya-Yala. Quito Ecuador. 1996, 468 PP.

Eede Joanna. Somos Uno. Un Homenaje a los Pueblos Indígenas. Impreso en China. Ediciones Blume. 2010, 224 PP.

Field C. Barry y otros. Economía Ambiental. Tercera Edición. Editorial McGRW-Hill Interamericano de España S.A.U. Madrid 2003, 556 PP.

Fontaine Guillaume y otros. Políticas Ambientales y Gobernabilidad en América Latina. Imprenta RisperGraf C.A. Quito, Ecuador 2007, 332 PP.

Galán Gómez y otros. 2006. El ciclo de proyecto de cooperación al desarrollo. Madrid, España. Editorial CYAN, proyectos y producciones editoriales S.A. 